\title{
Prepectoral breast reconstruction with complete anterior implant coverage using a single, large, square-shaped acellular dermis matrix
}

\section{Hyun Ki Hong}

Department of Plastic and Reconstructive Surgery, School of Medicine, Kyungpook National University

\section{Yun Hyun Kim}

Department of Plastic and Reconstructive Surgery, School of Medicine, Kyungpook National University Joon seok Lee

Department of Plastic and Reconstructive Surgery, School of Medicine, Kyungpook National University Jeeyeon Lee

Department of Surgery, School of Medicine, Kyungpook National University

\section{Ho Yong Park}

Department of Surgery, School of Medicine, Kyungpook National University

Jung Dug Yang ( $\nabla$ lambyang@knu.ac.kr)

Department of Plastic and Reconstructive Surgery, School of Medicine, Kyungpook National University

\section{Research Article}

Keywords: Prepectoral, Anterior coverage, Implant insertion

Posted Date: February 4th, 2022

DOI: https://doi.org/10.21203/rs.3.rs-1246299/v1

License: (1) This work is licensed under a Creative Commons Attribution 4.0 International License. Read Full License 


\section{Abstract}

\section{Background}

Several studies have discussed various methods of prepectoral direct-to-implant (DTI) breast reconstruction using an acellular dermis matrix (ADM) prosthesis to achieve full coverage; however, methods for anterior coverage are rarely reported. In this study, prepectoral DTI breast reconstruction with complete anterior implant coverage was performed using a square piece of ADM. This study aimed to introduce our prepectoral DTI technique and to identify its functional and cosmetic outcomes compared with existing subpectoral DTI techniques.

\section{Methods}

Our prospective cohort study focused on 29 patients (35 breasts) and 34 patients ( 35 breasts) who underwent breast reconstruction via subpectoral implant insertion (control) and anterior coverage prepectoral implant insertion, respectively. Postoperative complications were observed, and breast symmetry was evaluated using the Vectra $\mathrm{H} 2$ three-dimensional scanner. The modified Kyungpook National University Hospital Breast-Q (KNUH Breast-Q) was used to evaluate the patient's subjective satisfaction with the reconstruction and postoperative quality of life.

\section{Results}

There were no significant differences in complications in either group (i.e., seroma, skin necrosis, nippleareola complex necrosis, hematoma, capsular contracture, and infection). Statistically significantly better results were found in the anterior coverage group in the mean drain removal period. As compared with controls, the anterior coverage group had greater symmetry on three-dimensional scan, but this was not statistically significant. Subjective satisfaction and postoperative quality of life measured using the KNUH Breast-Q scale were not significantly different.

\section{Conclusions}

Considering its stability, faster recovery time, and cosmetic advantage, prepectoral breast reconstruction with anterior implant coverage using a single, large ADM is a good option in performing breast reconstruction with implant insertion in patients who have undergone mastectomy.

\section{Level of Evidence: Level II, Prospective cohort study.}

\section{Introduction}

Breast cancer is the most common cancer among women, and its prevalence increases each year (1). With the emergence of BRCA gene testing, prophylactic mastectomy is also becoming more common (2). Therefore, patients may wish to undergo breast reconstruction to resolve the defects caused by mastectomy and restore their cosmetic and functional aspects to normal (2). 
Generally, breast reconstruction can be performed using autologous tissue and prostheses. Direct-toimplant (DTI) breast reconstruction is a widely used prosthesis technique, and it is more common than autologous breast reconstruction. Thus, obtaining a deeper understanding of this method is imperative (3). Among DTI breast reconstructions, the subpectoral dual-plane technique has been widely used in the past; however, in recent years, prepectoral DTI breast reconstruction has become more popular $(2,4)$. Prepectoral DTI breast reconstruction has evolved from various methods and materials (4-7), and a material called acellular dermal matrix (ADM) has contributed enormously to its development $(2,4)$. Recently, various methods of prepectoral DTI breast reconstruction have been used to achieve implant coverage with an ADM. These methods have the ability to preserve the pectoralis major muscle $(8,9)$, which in turn reduces pain, animation deformity, and recovery time [1]. Furthermore, as compared with subpectoral DTI, prepectoral DTI is reported to have good cosmetic outcomes and a low occurrence of capsular contractures owing to the ease of adjusting the position of the inframammary fold (IMF) (1013).

Although prepectoral DTI breast reconstruction uses various methods and has numerous advantages in several aspects, there is no established consensus on the optimal choice of technique. A number of studies have been conducted on the methods of prepectoral DTI reconstruction using an ADM. Several techniques using a large piece of ADM to achieve full prosthesis coverage have been reported (14-16); our team has also reported this method by applying two ADMs crosswise (the two double-crossed ADM method) (9).

In addition to full prosthesis coverage, another method is to cover the prosthesis anteriorly (anterior coverage); however, few studies have been reported on this topic. As a method to achieve anterior coverage, our team performed prepectoral DTI breast reconstruction through complete anterior coverage of the implant using a single, large, square-shaped piece of the ADM. In this study, we aim to introduce our prepectoral DTI technique and compare its functional and cosmetic outcomes with those of the existing classic subpectoral DTI technique.

\section{Methods}

\section{Patients and data}

The Institutional Review Board of Kyungpook National University Hospital (Kyungpook national University Medical Center No. 2019-03-017) approved the study protocol. We performed a prospective cohort study to identify patients who had undergone single-stage prepectoral breast reconstruction with complete anterior implant coverage using a single, large, and square-shaped ADM and those who underwent surgery using the subpectoral DTI approach, with all surgeries performed by a single, senior surgeon. Between January 2019 and December 2020, The anterior coverage method was used in 35 breasts among 34 consecutive patients, and the subpectoral method was used in 35 breasts among 29 consecutive patients. 
We collected the following patient data: date of surgery, age at the time of surgery, body mass index, cancer staging, and the presence of radiation therapy (and timing, if present). Relevant complications and their specific types were also investigated.

\section{DTI breast reconstruction operative technique \\ Classic subpectoral DTI breast reconstruction}

The most suitable prosthesis was chosen based on the width and volume of the breasts and by measuring the weight of the excised mass immediately after mastectomy. To make a pocket for prosthesis insertion, pectoralis major muscle dissection was carefully performed by minimizing bleeding and damage to the surrounding tissue. For the portion that had insufficient coverage with an inferior part of muscle, the pocket for the prosthesis was completed using the ADM (Bellacell HD ${ }^{\text {TM }}$, HANSCARE, Seoul, South Korea).

\section{Prepectoral DTI breast reconstruction (anterior coverage)}

This anterior coverage technique uses a 16- · $16-\mathrm{cm}^{2}$ sheet of an ADM (Bellacell HD, HANSCARE), modified according to the size of the breast pocket and implant. The ADM was prepared on a separate sterile table followed by rehydration in iodine and normal saline according to the manufacturer's recommendations. Prepectoral pocket formation started by insetting the ADM over the pectoralis muscle in interrupted sutures using 2/0 Vicryl ${ }^{\circledR}$ (manufactured by Ethicon, Raritan, NJ, USA). To choose the appropriate implant size for breast reconstruction, an implant sizer was used. After inserting the appropriate implant sizer, the superior border of the ADM was fixed to the pectoralis major. Subsequently, while leaving the implant sizer in, the medial border of the ADM was fixed to the pectoralis major. When fixing the inferior aspect of the ADM, the location of the inferior aspect of ADM fixation was chosen to form a natural IMF by folding the ADM downward on the implant sizer. The ADM was sutured on the inferior aspect, leaving an opening on the lateral aspect to place the implant. The ADM in the inferior aspect was curved along the IMF perimeter to re-create the natural curvilinear contour of the breast with the patient repositioned in a $45^{\circ}$ upright position for a more realistic assessment of gravitational forces on the implant and soft-tissue envelope. After inserting the appropriate size of the prosthesis selected using the implant sizer, the lateral aspect was sutured according to the breast contour. After implant placement, the ADM provided complete anterior coverage of the prosthesis (Fig. 1). The skin-sparing mastectomy flaps were then tailored as necessary and closed in layers. Video 1 shows the surgical technique used for prepectoral breast reconstruction with complete anterior coverage of the implant using a single, large, square-shaped ADM.

\section{Clinical outcome}

Among patients who underwent subpectoral implant insertion and those who underwent prepectoral implant insertion with complete anterior coverage, we calculated the number and percentage of individuals who experienced seroma, linear skin necrosis, hematoma, capsular contracture, and infection, rippling. Complications were categorized into major (requiring rehospitalization or surgical treatment) and 
minor (can be treated through outpatient treatment) complications. Among minor complications, seroma was counted when the clinician observed enough fluid to require aspiration. The patients' subjective satisfaction with respect to reconstruction and quality of life (QoL) after surgery was evaluated using the modified Kyungpook National University Hospital Breast-Q (KNUH Breast-Q). Breast symmetry was measured postoperatively using the Vectra H2 (Canfield Scientific, Inc., Parsippany-Troy Hills, NJ, USA) three-dimensional (3D) scanner. Using this scanner to compare the level of symmetry in both groups, we measured the difference between the shortest distance of each sternal notch to the nipple (SN-N) and that of each IMF to the nipple (IMF-N), the difference in breast width between breasts, the difference in nipple to midline (N-M) between breasts, the difference in breast projection between the breasts, and the volume difference between breasts (Fig. 2). In addition, the cosmetic outcome was confirmed through gross photos captured during the postoperative follow-up period. All follow-up was performed by a single, senior surgeon.

\section{Statistical analysis}

Statistical significance was set at $p<0.05$. Statistical analysis was performed using IBM SPSS version 23.0 software (IBM Corp., Armonk, NY, USA). Chi-square test or Fisher's exact test were used for categorical variables, and a two-sample $t$ test was used for continuous variables.

\section{Results}

A total of 63 patients with breast cancer underwent skin-sparing mastectomy from January 2019 to December 2020. Our prospective cohort study was conducted on 29 patients (35 breasts) who underwent breast reconstruction through subpectoral implant insertion and 34 patients (35 breasts) who underwent breast reconstruction with prepectoral implant insertion using the complete anterior coverage method. Table 1 presents the demographic information of the patients. 
Table 1

Patient Characteristics ${ }^{a}$

\begin{tabular}{|c|c|c|c|}
\hline & $\begin{array}{l}\text { Subpectoral } \\
(n=29)\end{array}$ & $\begin{array}{l}\text { Prepectoral } \\
(n=34)\end{array}$ & value* \\
\hline No. of breasts & 35 & 35 & \\
\hline Age, years, mean \pm SD (range) & $43.8286 \pm 7.9428$ & $\begin{array}{l}47.2353 \pm \\
10.6716\end{array}$ & 0.0693 \\
\hline$\geq 0, N(\%)$ & $2(6.9 \%)$ & $0(0 \%)$ & \\
\hline$\geq 30, N(\%)$ & $4(13.8 \%)$ & $2(5.9 \%)$ & \\
\hline$\geq 40, N(\%)$ & $21(72.4 \%)$ & $20(58.8 \%)$ & \\
\hline$\geq 50, N(\%)$ & $2(6.9 \%)$ & $10(29.4 \%)$ & \\
\hline$\geq 60, \mathrm{~N}(\%)$ & $0(0 \%)$ & $2(5.9 \%)$ & \\
\hline$>70, \mathrm{~N}(\%)$ & $0(0 \%)$ & $0(0 \%)$ & \\
\hline $\mathrm{BMI}, \mathrm{kg} / \mathrm{m}^{2}$, mean $\pm \mathrm{SD}$ (range) & $22.2214 \pm 2.6532$ & $22.0161 \pm 2.3392$ & 0.3670 \\
\hline Excised mass weight $g$, mean \pm SD (range) & $\begin{array}{l}318.8571 \pm \\
122.2146\end{array}$ & $\begin{array}{l}286.4706 \pm \\
119.7632\end{array}$ & 0.0752 \\
\hline $\begin{array}{l}\text { Silicone implant volume, cc, mean } \pm \text { SD } \\
\text { (range) }\end{array}$ & $\begin{array}{l}346.7143 \pm \\
169.202\end{array}$ & $\begin{array}{l}284.4118 \pm \\
157.1419\end{array}$ & 0.0588 \\
\hline Cancer staging, $\mathrm{n}(\%)$ & & & 0.3494 \\
\hline 0 & $9(31.2 \%)$ & $7(20.5 \%)$ & \\
\hline I & $15(51.7 \%)$ & $17(50 \%)$ & \\
\hline$\|$ & $3(10.3 \%)$ & $9(26.6 \%)$ & \\
\hline III & $2(6.8 \%)$ & $1(2.9 \%)$ & \\
\hline IV & $0(0 \%)$ & $0(0 \%)$ & \\
\hline Chemotherapy, n (\%) & & & 0.1457 \\
\hline None & $22(75.8 \%)$ & $12(35.3 \%)$ & \\
\hline Neoadjuvant only & $0(0 \%)$ & $0(0 \%)$ & \\
\hline Neoadjuvant and adjuvant & $0(0 \%)$ & $0(0 \%)$ & \\
\hline Adjuvant only & $7(24.2 \%)$ & $22(64.7 \%)$ & \\
\hline Adjuvant hormone therapy & $18(62.5 \%)$ & $22(64.7 \%)$ & \\
\hline
\end{tabular}

${ }^{\mathrm{a} B M I}$, body mass index. 


\begin{tabular}{|llll|}
\hline & $\begin{array}{l}\text { Subpectoral } \\
(\mathbf{n = 2 9 )}\end{array}$ & $\begin{array}{l}\text { Prepectoral } \\
(\mathbf{n = 3 4 )}\end{array}$ & $\begin{array}{c}\boldsymbol{p} \\
\text { value* }\end{array}$ \\
\hline Radiotherapy, $\mathrm{n}(\%)$ & & & 0.7598 \\
\hline None & $25(86.2 \%)$ & $31(91.20 \%)$ & \\
\hline Neoadjuvant & $0(0 \%)$ & $0(0 \%)$ & \\
\hline Adjuvant & $4(13.8 \%)$ & $3(8.8 \%)$ & \\
\hline aBMl, body mass index. & & & \\
\hline
\end{tabular}

The complication rate was compared between the two groups, and the respective results of the subpectoral and anterior coverage groups are detailed below. The average follow-up period (mean \pm SD [range]) was $19.9 \pm 2.2274$ months in the subpectoral group and $16.3 \pm 8.8851$ months in the anterior coverage group. No major complications were observed in either group. The following minor complications were found in the subpectoral and anterior coverage groups, respectively: seroma (five patients [14.29\%] and three patients [8.57\%]), linear skin necrosis (five patients [14.29\%] and three patients [8.57\%]), hematoma (zero patients [0\%] in both groups), capsular contracture (one patient [2.86\%] and zero patients [0\%]), infection (one patient [2.86\%] and zero patients [0\%]), and rippling (zero patients $[0 \%]$ and two patients [5.71\%]). No statistical significance was found in any of the complications.

The mean period of drain removal (mean \pm SD [range]) was $10.5429 \pm 2.2274$ and $8.5588 \pm 2.5008$ days $(p<0.001)$ in the subpectoral and anterior coverage groups, respectively, with a statistically significant difference (Table 2).

Table 2. Complication rate

Number and percentage of patients who experienced seroma, linear skin necrosis, nipple-areola complex necrosis, hematoma, capsular contracture, and infection were recorded. Complications are mainly categorized as either major (i.e., requiring rehospitalization or surgical treatment) or minor (i.e., can be treated through outpatient treatment) complication. 


$\begin{array}{ll}\text { Subpectoral } & \text { Prepectoral } \quad p \text { value* } \\ (\mathbf{n}=\mathbf{2 9}) & (\mathrm{n}=\mathbf{3 4})\end{array}$

\begin{tabular}{llll} 
No. of breasts & 35 & 35 & \\
\hline $\begin{array}{l}\text { Mean follow-up period, months, mean } \pm \text { SD } \\
\text { (range) }\end{array}$ & $19.8771 \pm 5.9963$ & $\begin{array}{l}16.2931 \pm \\
8.8851\end{array}$ & 0.02750
\end{tabular}

\section{Major complication, $\mathrm{n}(\%)$}

\begin{tabular}{llll}
\hline Reoperation & $0(0 \%)$ & $0(0 \%)$ & \\
\hline Minor complication, $\mathbf{n}(\%)$ & & & \\
\hline Seroma & $5(14.29 \%)$ & $3(8.57 \%)$ & 0.2423 \\
\hline Linear skin necrosis & $5(14.29 \%)$ & $3(8.57 \%)$ & 0.1551 \\
\hline Hematoma & $0(0 \%)$ & $0(0 \%)$ & \\
\hline Capsular contracture & $1(4.76 \%)$ & $0(0 \%)$ & 0.1622 \\
\hline Infection & $1(4.76 \%)$ & $0(0 \%)$ & 0.1622 \\
\hline Rippling & $0(0 \%)$ & $2(5.71 \%)$ & 0.5188 \\
\hline No complication & $22(62.86 \%)$ & $25(71.43 \%)$ & 0.1741 \\
\hline $\begin{array}{l}\text { Mean drain removal period, days, mean } \mathbf{n} \text { SD } \\
\text { (range) }\end{array}$ & $11.1143429 \pm$ & $8.5588 \pm$ & $0.0275^{\star}$ \\
\hline
\end{tabular}

${ }^{*} p<0.05$

We evaluated the subjective satisfaction of patients regarding reconstruction and QoL after the surgery using the KNUH Breast-Q scale. No statistical significance was observed in any of the items (Table 3). 
Table 3

Patient satisfaction and quality of life evaluated using the modified KNUH University Hospital Breast-Q at 12 months postoperatively.

\begin{tabular}{|c|c|c|c|c|}
\hline & $\begin{array}{l}\text { Very } \\
\text { satisfied }\end{array}$ & $\begin{array}{l}\text { Subpectoral } \\
(n=29)\end{array}$ & $\begin{array}{l}\text { Prepectoral } \\
(n=34)\end{array}$ & value* $^{p}$ \\
\hline $\begin{array}{l}\text { 1. Overall, are you satisfied with your breast } \\
\text { reconstruction? }\end{array}$ & 5 & 4.3 & 4.3 & 0.125 \\
\hline $\begin{array}{l}\text { 2. Are you satisfied with breast symmetry } \\
\text { achieved after reconstruction? }\end{array}$ & 5 & 4.0 & 4.1 & 0.482 \\
\hline $\begin{array}{l}\text { 3. Are you satisfied with the size of your breast } \\
\text { after reconstruction? }\end{array}$ & 5 & 4.2 & 4.3 & 0.247 \\
\hline $\begin{array}{l}\text { 4. Are you satisfied with the shape of your breast } \\
\text { after reconstruction? }\end{array}$ & 5 & 3.9 & 4.0 & 0.542 \\
\hline $\begin{array}{l}\text { 5. Are you satisfied with how your breasts feel } \\
\text { after reconstruction? }\end{array}$ & 5 & 4.2 & 4.0 & 0.127 \\
\hline $\begin{array}{l}\text { 6. Are you satisfied with the level of pain you had } \\
\text { to endure after reconstruction? }\end{array}$ & 5 & 4.3 & 4.2 & 0.424 \\
\hline $\begin{array}{l}\text { 7. Are you satisfied with the scar resulted after } \\
\text { breast reconstruction? }\end{array}$ & 5 & 3.7 & 3.9 & 0.542 \\
\hline $\begin{array}{l}\text { 8. Have you experienced a loss of confidence or } \\
\text { self-esteem after breast reconstruction? }\end{array}$ & 5 & 4.1 & 4.3 & 0.984 \\
\hline $\begin{array}{l}\text { 9. Are you satisfied with your sexual } \\
\text { attractiveness after breast reconstruction? }\end{array}$ & 5 & 4.1 & 4.2 & 0.654 \\
\hline Total & 55 & 44.8 & 45.2 & 0.214 \\
\hline
\end{tabular}

We measured postoperative breast symmetry using the Vectra H2 3D scanner. In the subpectoral and prepectoral groups, the difference between the shortest $\mathrm{SN}-\mathrm{N}$ distances (mean $\pm \mathrm{SD}$ [range]) was 0.7783 \pm 0.6510 and $0.5882 \pm 0.5944 \mathrm{~cm}(p=0.1048)$, between the shortest distances from the IMF line to the nipple was $0.8 \pm 0.4802$ and $0.7176 \pm 1.1269 \mathrm{~cm}(p=0.3482)$, between breast widths was $1.0229 \pm$ 0.7670 and $0.7471 \pm 0.7886 \mathrm{~cm}(p=0.0728)$, between the shortest distances from the nipple to the midline $(\mathrm{N}-\mathrm{M})$ was $0.9971 \pm 0.5090$ and $0.9441 \pm 0.7468 \mathrm{~cm}(p=0.3662)$, between breast projection was $0.4143 \pm 0.3805$ and $0.4853 \pm 0.3322 \mathrm{~cm}(p=0.0690)$, and between breast volume was $38.3814 \pm$ 28.2869 and $36.9559 \pm 27.6897 \mathrm{~cm}(p=0.4166)$, respectively. Although the overall difference was lower in the prepectoral DTI patient group than in the subpectoral DTI patient group, no statistical significance was observed in either group regarding all items (Table 4).

Table 4. Symmetry measurement using 3D scanner 
The difference between the shortest distance of each sternal notch to the nipple (SN-N), that of each IMF to the nipple (IMF-N), the difference in projection between both breasts, and the volume difference between both breasts were measured using the Vectra H2 (Canfield Scientific, Inc.) 3D scanner to compare the level of symmetry between both groups. ${ }^{\text {a }}$

$\begin{array}{lll}\text { Subpectoral } & \text { Prepectoral } & p \\ (\mathbf{n}=\mathbf{2 9}) & (\mathrm{n}=\mathbf{3 4}) & \text { value* }^{*}\end{array}$

No. of breasts

35

35

$\mathrm{SN}-\mathrm{N}$ distance difference, $\mathrm{cm}$, mean $\pm \mathrm{SD}$ (range)
$0.7783 \pm 0.651$
$0.5882 \pm 0.5944$
0.1048

IMF-N distance difference, $\mathrm{cm}$, mean $\pm \mathrm{SD}$ (range)

$0.8 \pm 0.4802 \quad 0.7176 \pm 1.1269$

0.3482

Breast width difference, $\mathrm{cm}$, mean \pm SD (range)

$\mathrm{N}$-M distance difference, $\mathrm{cm}$, mean $\pm \mathrm{SD}$ (range)

Breast projection difference, $\mathrm{cm}$, mean $\pm \mathrm{SD}$ (range)
Breast volume difference, $c c$, mean \pm SD (range)
$38.3814 \pm$
28.2869
$36.9559 \pm$
27.6897
0.4166

$0.7471 \pm 0.7886 \quad 0.0728$

$0.9971 \pm 0.509 \quad 0.9441 \pm 0.7468 \quad 0.3662$

aSN-N, sternal notch to nipple; IMF-N, inframammary fold line to nipple; $\mathrm{N}-\mathrm{M}$, nipple to midline. ${ }^{\star} p<0.05$

\section{Discussion}

Among breast reconstruction methods using a prosthesis, prepectoral breast reconstruction is acknowledged for its stability and cosmetic outcomes. Sigalove et al reported that prepectoral breast reconstruction is a safe method with predictable results (17). In addition, several papers have reported its excellent postoperative outcomes, such as reducing pain and animation deformity owing to the preservation of the pectoralis major (18-21).

Several previous studies have discussed prepectoral DTI breast reconstruction. The study by Reitsamer et al described the full coverage of the prosthesis using porcine ADM Strattice (LifeCell Corporation, Bridgewater, NJ, USA) (4), whereas Vidya et al reported beneficial results from a method that fully covers the prosthesis using Braxon ${ }^{\circ}$, a single large ADM (22). Similarly, our team reported outcomes of prepectoral DTI breast reconstruction with complete implant coverage using a double-crossed ADM (9). Most prepectoral DTI breast reconstruction using ADM described in several studies used ADM for full coverage of the implant (14-16). 
Some studies have also reported the anterior coverage method, wherein only the anterior part of the implant is covered. Kyle et al introduced a technique for anterior coverage using a method to sling AlloDerm ${ }^{\text {TM }}$ partially through the dual-plane technique (23). Ayesha et al and Yang et al reported methods involving complete anterior coverage of an implant $(24,25)$, but only a few studies have discussed a method that completely covers the anterior part of the ADM (i.e., complete anterior coverage).

Our team performed prepectoral DTI breast reconstruction, in which the implant is inserted into a pocket, to achieve complete anterior coverage. This was made by fixing a single, large, square-shaped ADM to the pectoralis major. Our study aimed to determine the functional and cosmetic outcomes of our prepectoral DTI method and compare the outcomes with those of the classic subpectoral DTI technique.

We compared postoperative outcomes between patients who underwent the subpectoral technique and those who underwent the prepectoral technique. We also evaluated complication rates, patient satisfaction using the KNUH Breast-Q scale, and symmetry using the Vectra H2 3D scanner. No significant difference in major and minor (i.e., seroma, linear skin necrosis, hematoma, capsular contracture, infection, and rippling) complications was observed between the two groups. However, the mean drain removal period was $10.5429 \pm 2.2274$ and $8.5588 \pm 2.5008$ days $(p<0.001)$ in the subpectoral and anterior coverage groups, respectively. This implies that it takes less time to recover when using the anterior coverage technique versus the subpectoral technique. The hospitalization period is shortened, and the patient can return to daily life faster because of early discharge. Furthermore, the anterior coverage technique had noninferior stability and faster recovery time than the subpectoral technique did.

Moreover, it has been reported that the percentage of skin necrosis may be high when conducting prepectoral reconstruction along with mastectomy skin flap (1). In our study, a lower percentage of linear skin necrosis was observed in prepectoral reconstruction using the anterior coverage method versus the subpectoral method, but its statistical significance was not verified. This is thought to have an effect, as surgery was performed only for patients with an indication after confirming that the thickness of the mastectomy flap and the perfusion state were appropriate for prepectoral reconstruction. In addition, it is thought that because our surgical method covers the anterior surface of the implant after spreading the ADM wide, the relatively good adhesion between the skin flap and the ADM might also have had an effect.

One case $(2.86 \%)$ of capsular contracture occurred in the subpectoral DTI group. This patient received postmastectomy radiation therapy after mastectomy and subpectoral DTI. Two patients received postmastectomy radiation therapy in the anterior coverage group, but no capsular contracture occurred in these patients (Fig. 3).

Infection occurred in one patient (2.86\%) in the subpectoral patient group and in none (0\%) of the patients in the anterior coverage group. Despite our compliance with the hospital's infection prevention protocol when performing breast reconstruction through DTI (26), occasional cases of infection still occur. In these cases, our team immediately administered empirical antibiotics and referred the patient to other 
departments to quickly diagnose and establish a treatment plan so that proactive surgical intervention could be considered alongside appropriate drug therapy (26). In the single case with infection in this study, broad-spectrum antibiotics were administered immediately upon appearance of symptoms. Salvage reoperation was not performed, as the symptoms of infection subsided within a short time.

A previous study found that implant visibility and rippling occur frequently after breast reconstruction with prepectoral DTI (9). In this study, rippling occurred slightly more frequently in the anterior coverage group than in the subpectoral DTI group (two patients [5.71\%] vs. zero patients [0\%]). As a patient group for prepectoral DTI, an indication is set as a group of patients who had undergone skin-sparing or nipplesparing mastectomy; flap quality (thickness, vascularity) should be confirmed, and it is advantageous to exclude from the indication for preoperative radiotherapy history, current smokers, and patients with uncontrollable diabetes mellitus in which the skin flap may not be in good condition (9).

We found no significant difference between the two groups in the KNUH Breast-Q scale, which evaluated subjective satisfaction with breast reconstruction and QoL after surgery. Furthermore, no significant differences were found in symmetry measurement using the Vectra H2 3D scanner. The Vectra H2 3D scanner enables the measurement of the $\mathrm{SN}-\mathrm{N}$ distance, IMF-N difference, breast width, $\mathrm{N}-\mathrm{M}$ distance, breast projection difference, and breast volume of patients (Fig. 2). Yan Yang et al reported measurements of breast symmetry using surface anatomy (27). Although there are some discrepancies between their surface anatomy parameters and those of our study, we did not experience difficulties measuring breast symmetry using our parameters.

According to our results, there was no statistically significant difference in symmetry measured by the 3D scanner, but a smaller difference was observed in the anterior coverage group in all items. This represents the superiority of anterior coverage in adjusting breast symmetry during breast reconstruction, which is in line with the findings of existing studies claiming that prepectoral breast reconstruction can yield superior cosmetic outcomes. The superiority in cosmetic outcome can be seen in the gross photos captured in patients with anterior coverage during their follow-up (Figs. 3 and 4).

During prepectoral DTI breast reconstruction with anterior coverage, all aspects of ADM are fixed to the pectoralis muscle. In particular, when fixing the inferior aspect of ADM, it is made to form a natural IMF by folding the ADM downward into the implant sizer (Fig. 1); this may improve cases of ptotic breasts. Compared with the prepectoral DTI technique through full wrapping, the prepectoral DTI technique with anterior coverage has an effect of reducing the dead space between the skin flap and ADM. Unlike full wrapping, wherein implants are wrapped based on the shape of the ADM, the anterior coverage technique takes into consideration the natural breast shape, and thus the ADM is spread over the implant, and the anterior surface of the implant is covered. The outcome of complications such as seroma were better with the anterior coverage method than with the full wrapping method, although these findings require verification in further studies.

Despite its prospective cohort study design, one limitation of this study is the small number of patients in both groups, which hindered statistical verification. In addition, the superiority of the anterior coverage 
method could have been better demonstrated by comparing it with prepectoral breast reconstruction methods other than the subpectoral method. Finally, the relatively short follow-up period in some patients is considered to be another limitation.

Regardless of these limitations, our findings demonstrate that anterior coverage-based breast reconstruction had superior outcomes over the existing subpectoral implant-based reconstruction in terms of faster recovery time and breast symmetry.

\section{Conclusions}

We report the superiority of the complete anterior coverage implant method using a single, large, squareshaped ADM in breast reconstruction through DTI after mastectomy. The superiority of this technique is based on its stability, faster recovery time, and cosmetic outcomes. Prepectoral breast reconstruction with anterior implant coverage using a single, large ADM is a good option for performing breast reconstruction with implant insertion in patients who have undergone mastectomy.

\section{Abbreviations}

DTI : Direct-to-implant

ADM : Acellular dermis matrix

KNUH : Kyungpook national university hospital

IMF : Inframammary fold

QoL : Quality of life

SN-N : Sternal notch to the nipple

IMF-N : Inframammary fold to nipple

N-M : Nipple to midline

\section{Declarations}

Availability of data and materials: The data that support the findings of this study are available on request from the corresponding author. The data are not publicly available due to privacy or ethical restrictions.

Acknowledgements: We would like to thank Enago (www.enago.com) for English language editing

Funding: The authors received no grants, equipment or funding for this study. 


\section{Affiliations}

Department of Plastic and Reconstructive Surgery, School of Medicine, Kyungpook National University, 130 Dongdeokro, Jung-gu, Daegu 41944, Korea

Hyun Ki Hong, Yun Hyun Kim, Joon seok Lee, Jung Dug Yang

Department of Surgery, School of Medicine, Kyungpook National University, 130 Dongdeokro, Jung-gu, Daegu 41944, Korea

Jeeyeon Lee, Ho Yong Park

Contributions: $\mathrm{HKH}$ is a surgeon in plastic surgery training. He has previously written several papers, and this paper has also been written under the guidance of the corresponding author. There are a total of 6 authors of this paper, and YHK provided administrative support, data collection, and analysis. JSL provided study data and patients, and collected and analyzed data. JL provided research data and patients, and wrote a manuscript. HYP provided administrative support and wrote manuscripts. The corresponding author, JDY, decided and designed the research concept and wrote a manuscript. All authors participated in manuscript writing, and all authors confirmed and approved the final manuscript.

Corresponding author: Correspondence to Jung Dug Yang

Ethics approval and consent to participate: All procedures followed were in accordance with the ethical standards of the responsible committee on human experimentation (institutional and national) and with the Helsinki Declaration of 1964 and later versions. The Institutional Review Board of Kyungpook National University Hospital (Kyungpook National University Medical Center No. 2019-03-017) approved the study and the requirement for informed consent was waived.

Consent for publication: Not applicable

Competing interests: The authors declare that they have no competing interests.

\section{References}

1. Momeni A, Remington AC, Wan DC, Nguyen D, Gurtner GC. A Matched-Pair Analysis of Prepectoral with Subpectoral Breast Reconstruction: Is There a Difference in Postoperative Complication Rate?. Plast Reconstr Surg. 2019;144(4):801-807.

2. Lee JS, Park E, Lee JH, et al. A prospective comparison study of early functional outcomes after implant-based breast reconstruction: subpectoral versus prepectoral technique. Ann Palliat Med. 2021;10(3):2520-2529.

3. Albornoz CR, Bach PB, Mehrara BJ, et al. A paradigm shift in U.S. Breast reconstruction: increasing implant rates. Plast Reconstr Surg. 2013;131(1):15-23. 
4. Reitsamer R, Peintinger F. Prepectoral implant placement and complete coverage with porcine acellular dermal matrix: a new technique for direct-to-implant breast reconstruction after nipplesparing mastectomy. J Plast Reconstr Aesthet Surg. 2015;68(2):162-167.

5. Ibrahim AM, Koolen PG, Ashraf AA, et al. Acellular Dermal Matrix in Reconstructive Breast Surgery: Survey of Current Practice among Plastic Surgeons. Plast Reconstr Surg Glob Open. 2015;3(4):e381.

6. Gandhi A, Barr L, Johnson R. Bioprosthetics: changing the landscape for breast reconstruction?. Eur J Surg Oncol. 2013;39(1):24-25.

7. Macadam SA, Lennox PA. Acellular dermal matrices: Use in reconstructive and aesthetic breast surgery. Can J Plast Surg. 2012;20(2):75-89.

8. Downs RK, Hedges K. An Alternative Technique for Immediate Direct-to-Implant Breast Reconstruction-A Case Series. Plast Reconstr Surg Glob Open. 2016;4(7):e821.

9. Lee JS, Kim JS, Lee JH, et al. Prepectoral breast reconstruction with complete implant coverage using double-crossed acellular dermal matrixs. Gland Surg. 2019;8(6):748-757.

10. Parikh PM, Spear SL, Menon N, Reisin E 20: Immediate breast reconstruction with tissue expanders and AlloDerm. Plast Reconstr Surg. 2006;118(4):18.

11. Basu CB, Leong M, Hicks MJ. Acellular cadaveric dermis decreases the inflammatory response in capsule formation in reconstructive breast surgery. Plast Reconstr Surg. 2010;126(6):1842-1847.

12. Vardanian AJ, Clayton JL, Roostaeian $\mathrm{J}$, et al. Comparison of implant-based immediate breast reconstruction with and without acellular dermal matrix. Plast Reconstr Surg. 2011;128(5):403e410e.

13. Kim JYS, Davila AA, Persing S, et al. A meta-analysis of human acellular dermis and submuscular tissue expander breast reconstruction. Plast Reconstr Surg. 2012;129(1):28-41.

14. Vidya R, Iqbal FM. A Guide to Prepectoral Breast Reconstruction: A New Dimension to Implant-based Breast Reconstruction. Clin Breast Cancer. 2017;17(4):266-271.

15. Downs RK, Hedges K. An Alternative Technique for Immediate Direct-to-Implant Breast Reconstruction-A Case Series. Plast Reconstr Surg Glob Open. 2016;4(7):e821.

16. Cattelani L, Polotto S, Arcuri MF, Pedrazzi G, Linguadoca C, Bonati E. One-Step Prepectoral Breast Reconstruction With Dermal Matrix-Covered Implant Compared to Submuscular Implantation: Functional and Cost Evaluation. Clin Breast Cancer. 2018;18(4):e703-e711.

17. Sigalove S, Maxwell GP, Sigalove NM, et al. Prepectoral Implant-Based Breast Reconstruction: Rationale, Indications, and Preliminary Results. Plast Reconstr Surg. 2017;139(2):287-294.

18. Sbitany H. Important Considerations for Performing Prepectoral Breast Reconstruction. Plast Reconstr Surg. 2017;140(6S Prepectoral Breast Reconstruction):7S-13S.

19. Sbitany H, Piper M, Lentz R. Prepectoral Breast Reconstruction: A Safe Alternative to Submuscular Prosthetic Reconstruction following Nipple-Sparing Mastectomy. Plast Reconstr Surg. 2017;140(3):432-443. 
20. Salibian AA, Frey JD, Choi M, Karp NS. Subcutaneous Implant-based Breast Reconstruction with Acellular Dermal Matrix/Mesh: A Systematic Review. Plast Reconstr Surg Glob Open. 2016;4(11):e1139.

21. Bernini M, Calabrese C, Cecconi L, et al. Subcutaneous Direct-to-Implant Breast Reconstruction: Surgical, Functional, and Aesthetic Results after Long-Term Follow-Up. Plast Reconstr Surg Glob Open. 2016;3(12):e574.

22. Vidya R, Masià J, Cawthorn S, et al. Evaluation of the effectiveness of the prepectoral breast reconstruction with Braxon dermal matrix: First multicenter European report on 100 cases. Breast $\mathrm{J}$. 2017;23(6):670-676.

23. Chepla KJ, Dagget JR, Soltanian HT. The partial AlloDerm sling: reducing allograft costs associated with breast reconstruction. J Plast Reconstr Aesthet Surg. 2012;65(7):924-930.

24. Khan A, Tasoulis MK, Teoh V, Tanska A, Edmonds R, Gui G. Pre-pectoral one-stage breast reconstruction with anterior biological acellular dermal matrix coverage. Gland Surg. 2021;10(3):1002-1009.

25. Yang JY, Kim CW, Lee JW, Kim SK, Lee SA, Hwang E. Considerations for patient selection: Prepectoral versus subpectoral implant-based breast reconstruction. Arch Plast Surg. 2019;46(6):550-557.

26. Yeo H, Lee D, Kim JS, et al. Strategy for salvaging infected breast implants: lessons from the recovery of seven consecutive patients. Arch Plast Surg. 2021;48(2):165-174.

27. Yang Y, Mu D, Xu B, et al. An Intraoperative Measurement Method of Breast Symmetry Using ThreeDimensional Scanning Technique in Reduction Mammaplasty. Aesthetic Plast Surg. 2021;45(5):2135-2145.

\section{Figures}

\section{Figure 1}

Surgical technique of anterior coverage

(a) After inserting an appropriate implant sizer, the acellular dermis matrix (ADM) was sutured from the superior margin. (b) While retaining the implant sizer, the medial border of the ADM was fixed to the pectoralis major. (c) The ADM was fixated by folding the ADM downward into the implant sizer to form a natural inframammary fold, after which the implant sizer was removed. (d) The inferior margin of the ADM was sutured, leaving an opening on the lateral aspect to place the implant. (e) Insertion of the implant. (f) After implant placement, the ADM provides total anterior coverage of the prosthesis.

\section{Figure 2}


Measurement of symmetry parameters using the Vectra H2 3D scanner.

(A) Anterior-posterior view. (a) Sternal notch to nipple (SN-N) distance. (b) Inframammary fold line to nipple (IMF-N) difference. (c) Breast width. (d) Nipple to midline (N-M) distance. (e) Breast volume.

(B) Craniocaudal view. (f) Breast projection difference.

\section{(A)

\section{Figure 3}

Breast reconstruction with prepectoral implant insertion by anterior coverage ( $F / 39)$. (a, b) Preoperative appearance. (c, d) Postoperative appearance at 1.5 years.

\section{Figure 4}

Breast reconstruction with prepectoral implant insertion via anterior coverage (F/51).

(a) Preoperative appearance. (b) Postoperative appearance at 5.5 months (immediately after postmastectomy radiotherapy). (c) Postoperative appearance at 12 months (6.5 months after postmastectomy radiotherapy).

\section{Supplementary Files}

This is a list of supplementary files associated with this preprint. Click to download. 
- Videoonline.mp4

Page 18/18 